\title{
Three-dimensional measurement of rolling in tetraplegic patients
}

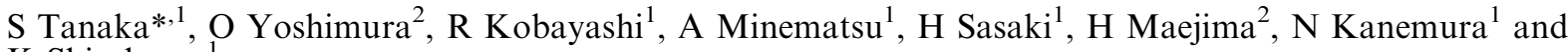 \\ K Shirahama \\ ${ }^{1}$ Graduate School of Medical Sciences, Hiroshima University School of Medicine, Hiroshima, Japan; ${ }^{2}$ Institute of \\ Health Sciences, Hiroshima University School of Medicine, Hiroshima, Japan
}

\begin{abstract}
Objective: To investigate the differences between traumatic tetraplegic patients who can roll and those who cannot.

Design: Motion analysis using 3-dimensional measurement.

Setting: Rehabilitation centers in southwestern Japan.

Participants: Nineteen male participants, all of whom had traumatic C6 complete injury.

Methods: We used an electromagnetic device to examine the degree of spinal movement in axial rotation during rolling (shifting from supine to side lying). This system (3-Space Win) measures the position and orientation of sensors in space. Two sensors were mounted on a subject over the spinous process of T1 and L5.

Results: The spinal rotation of patients who could not roll was significantly lower than that of patients who could roll. (The average rotation of non-rollers was $31.5 \pm 17.5^{\circ}$, while the average rotation of rollers was $66.3 \pm 17.3^{\circ}$ ). In this study, there were no statistically significant differences in the members of the two groups in terms of age, height, weight or time after injury.

Conclusion: Rolling requires greater and adequate flexibility in the back of tetraplegic patients.

Spinal Cord (2000) 38, 683-686
\end{abstract}

Keywords: spinal cord injury; tetraplegia; motion analysis; rolling

\section{Introduction}

The possibility of independence for tetraplegic patients in activities of daily living (ADL) depends on the neurological level of the activity. ${ }^{1,2}$ With regard to rolling, C5 tetraplegic patients have difficulty, but C7 tetraplegic patients are much more capable. ${ }^{3}$ Some C6 complete tetraplegics can roll, while some cannot. Differences in the degree of mobility among tetraplegic patients with similar levels of injury have been noted on occasion. The degree of disability varies most widely among C6 tetraplegic patients in comparison to patients injured at other neurological levels. ${ }^{4}$ The purpose of this study is to investigate the differences between C6 traumatic tetraplegic patients (complete injury) who can roll and those who cannot using 3dimensional measurement.

The mobility of the spine seems to play a key part in rolling, but this quantity has not been studied directly in the past. This is due, in part, to the technical problem of measuring the movement of

*Correspondence: S Tanaka, Graduate School of Medical Sciences, Hiroshima University School of Medicine, 1-2-3 Kasumi, Minami-ku, Hiroshima 734-8551, Japan markers, which are hidden behind the body when a subject lays supine. In the present study, we used an electromagnetic device to solve this problem.

\section{Subjects}

Nineteen male subjects participated in the study after providing informed consent. All subjects were diagnosed with complete C6 tetraplegia and had completed their hospital based medical rehabilitation. The median period post injury for the subjects was 59.5 (range 21-381) months. Table 1 reports the age, height, weight and time post injury for the subject population.

\section{Methods}

'Rolling' in this study is defined as motion from a supine position to a side lying position using only the swinging of the upper limbs for assistance (ie, without using any tools such as a fence of the bed). If a patient was able to perform this motion (from a supine position to a side lying position), it would then be easier for the patient to roll from a side lying to a 
Table 1 Physical characteristics of study participants (C6)

\begin{tabular}{llcc}
\hline & & Roller & Non-Roller \\
\hline $\mathrm{N}$ & & 10 & 9 \\
Age & Mean & 27.3 & 35.7 \\
& SD & 7.2 & 15.5 \\
Height (cm) & Mean & 170.4 & 171.7 \\
& SD & 4.8 & 3.9 \\
Weight (kg) & Mean & 53.1 & 55.0 \\
& SD & 10.7 & 10.9 \\
Time post injury & Mean & 94.4 & 115.6 \\
(month) & SD & 83.6 & 118.3 \\
\hline
\end{tabular}

prone position, because the weight of the upside upper limb and the upper part of the body could be used to help carry out the motion. A patient used a momentum that made him move from a supine position to a side lying position continuously. The movement from supine to side lying seemed to be important in preventing the development of sores during bed rest, even if the patient was not able to fully roll over (from the supine to the prone position).

\section{Devices}

An electromagnetic device was used to examine the degree of spinal movement used for axial rotation during rolling. This system (3-Space Win: Polhemus, USA) measured the position and orientation of sensors in space. Two sensors were mounted on the subject over the spinous process of T1 and L5 using doublesided tape and elastic tape. Even if the sensors were behind the body in the supine position, their movements could be tracked. The data sampling rate was $60 \mathrm{~Hz}$. The error was less than $0.8 \mathrm{~cm}$ in measuring the position and $0.15^{\circ}$ in measuring the orientation within a radius of $75 \mathrm{~cm} .^{5}$

\section{Measurement}

Spinal axial rotation during rolling The subjects attempted to roll three times from a supine position to a side lying position. If a patient performed differently on one side or the other (eg, if a patient could successfully roll to one side but not to the other), axial rotation was measured for both directions. Otherwise, it was measured for only one direction (towards left side); (25 cases: 11 Rollers, 14 Nonrollers).

Passive rotation towards the direction a subject rolls The passive range of motion (ROM) was measured with a goniometer using the standard method. One of the examiners held both of the subject's anterior superior iliac spines (ASIS) so that they did not move. To minimize error, the examiner who measured, the physiotherapist who led the passive movement, and the physiotherapist who held the ASIS were the same for all subjects. Spinal rotation was measured using an electromagnetic device at the same time as measurement of the passive ROM.

To check the swing of the upper extremities, a video camera was fixed over the subject's head.

\section{Analysis}

Analysis of the patients' characteristics Nine of the subjects could not roll to either side ('Non-rollers'). The other ten could roll to at least one side ('Rollers'). The results were then analyzed according to the following subject characteristics: age, height, weight, and time post injury (Table 1).

Analysis of spinal rotation The axes of the spatial coordinates are as shown in Figure 1. Axial rotation was calculated around the $\mathrm{y}$-axis between the two sensors (TH1, L5). The angle at the start position was set to be $0^{\circ}$, then the relative rotation between the sensors was calculated. Each subject's maximum movement for each trial was used to compile the mean results for all subjects.

\section{Statistical processing}

Following processing, statistical analysis was used to compare the values of the Rollers and the Non-rollers. First, normality was analyzed. If normality was present, the unpaired $t$-test (Student or Welch) was used. If not, Mann-Whitney's U-test was used. Differences were deemed to be statistically significant at $P<0.05$.

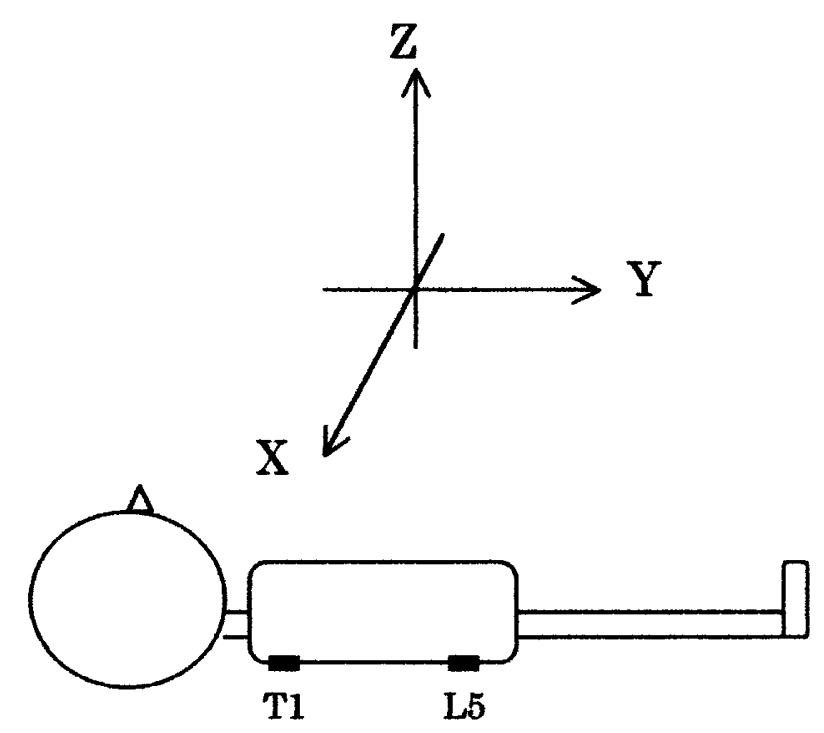

Figure 1 The axes of the spatial coordinates 


\section{Results}

There were no statistically significant differences in the properties of members of the two groups: age, height, weight, and time post injury in this study (Table 1).

The axial rotation upon rolling (peak spinal rotation upon rolling) was significantly lower in patients who could not roll than in patients who could roll. The Rollers' average rotation was $66.3+17.3^{\circ}$, and the Non-Rollers' average rotation was $31.5 \pm 17.5^{\circ}(P<0.0001)$ (Figure 2$)$.

The two groups exhibited no significant differences upon passive rotation according to both the goniometer and the electromagnetic device (Table 2, Figure $3)$.

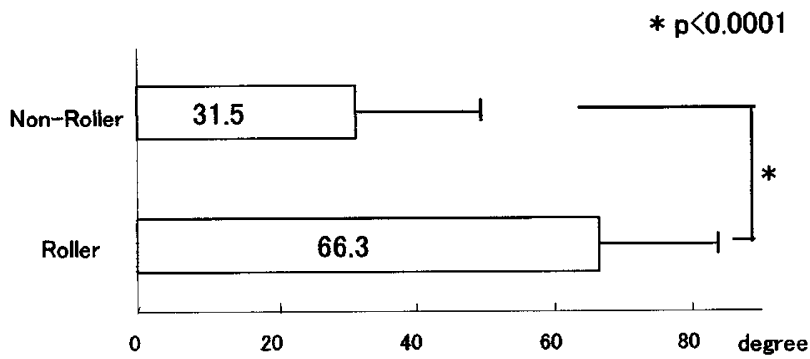

Figure 2 Spinal rotation upon rolling

Table 2 Axial rotation

\begin{tabular}{llcc}
\hline & & Roller & $\begin{array}{c}\text { Non- } \\
\text { Roller }\end{array}$ \\
\hline $\mathrm{N}$ & & 11 & 14 \\
& & & \\
Spinal axial rotation during & Mean & 66.3 & 31.5 \\
$\quad$ rolling (degree) & SD & 17.3 & 17.5 \\
Passive ROM (degree) & Mean & 84.0 & 83.0 \\
$\quad$ with a goniometer & SD & 7.4 & 17.2 \\
Passive ROM (degree) & Mean & 68.8 & 63.1 \\
$\quad$ with an electromagnetic device & SD & 14.3 & 10.3 \\
\hline
\end{tabular}

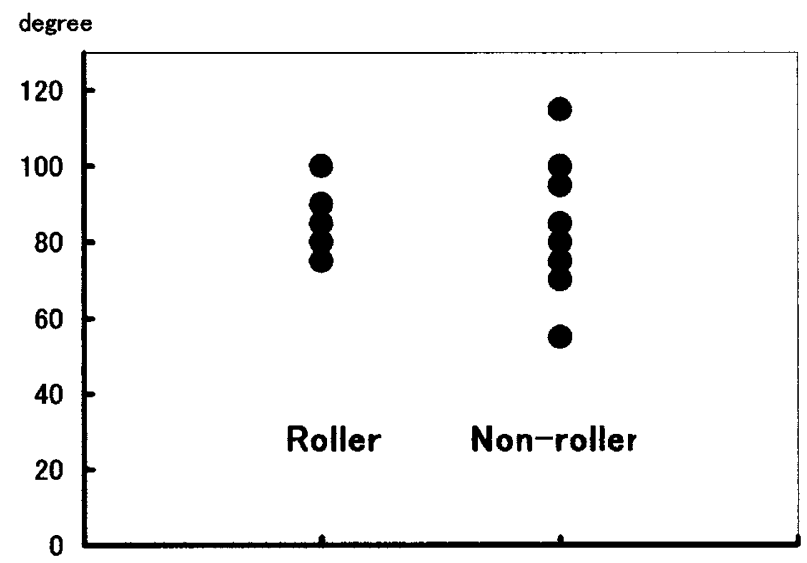

Figure 3 Passive ROM (by a goniometer)

\section{Discussion}

Axial rotation in motion (rolling)

In this study we found a significant difference in the degree of axial rotation upon rolling between the Rollers and the Non-rollers. Our previous study, with the same procedure on healthy individuals, the average angle of trunk rotation during usual rolling was $33.6 \pm 20.3^{\circ} .{ }^{6}$ This value was similar to that of the Non-rollers in the present study. This finding suggests that, for tetraplegics, rolling requires more flexibility in spinal motion than it does for healthy individuals.

The difference in the degree of spinal rotation needed for rolling between tetraplegic Rollers and healthy men showed that even if the patients had been able to roll before being injured, we could not conclude that they would have enough flexibility to roll as tetraplegics.

Recently, a number of cases of elderly tetraplegics have been reported. ${ }^{7,8}$ In these cases, less flexibility in the trunk seems to cause difficulty in rolling.

Range of motion (ROM) in trunk (in passive) Tetraplegics have few residual muscles, and the few that they have are generally weaker than those of normal subjects. It therefore becomes important for tetraplegics to roll as efficiently as possible in order to maximise what little muscle power they have. Healthy individuals can begin to roll from any part of the body, eg, head, shoulder girdle, pelvic girdle, etc. However, standard rolling for tetraplegic patients is uniform: the tetraplegic must make the head, neck and shoulder girdle rotate using a few residual muscles, then direct his or her power through the trunk and pelvic area down to the lower extremities.

The higher a tetraplegic patient's level, the less muscle he or she is able to use as a power source in moving. Therefore, motion becomes more difficult. ${ }^{9}$ Tetraplegics whose level is $\mathrm{C} 6$ or higher cannot swing their upper limbs sufficiently to roll. Paraplegic patients with thoracic or lumbar cord injury cannot push against the floor, either, to assist them in rotating, and therefore require more rotatomomentum in the upper part of the body in order to roll. For these reasons, substantial ROM of the spine is required.

We have had some experience with poor flexibility of a patient's spine causing the patient difficulty in rolling. However, this was not associated with any significant differences in the ability to roll related to trunk flexibility (passive ROM). One possible reason for this finding is that most of the subjects of this study were young (average age: $31.3 \pm 12.3$ years). There were few older subjects on which to study the lack of flexibility that results from advancing past middle age. This absence of older subjects might cause the lack of a significant difference in ROM between the two groups. 
Another reason for the lack of difference was the presence of several subjects who exhibited too much flexibility in the trunk to be able to roll. Those subjects' pelvises could not be dragged by swinging their upper limbs, and so they failed to finish the rolling motion (trunk ROM ranges were $75-100^{\circ}$ for Rollers and $55-115^{\circ}$ for Non-Rollers). A certain level of flexibility, therefore, was seen to help subjects to roll, while too great a level prevented subjects from spreading the rotation of the upper limbs to the pelvis. The evidence that there were no significant differences when spinal rotation was measured passively would suggest a functional capacity. Having excess of range of motion (passive rotation) did not mean a better rolling capacity. Increased passive rotatory movement might actually impede the transfer of angular momentum to the lower spine and pelvis. Therefore some range stiffness might be an important factor in the transfer of momentum along the spine (especially in the lower part). Further investigation is required to ascertain the appropriate ROM to facilitate.

As a society ages, the number of spinal cord injury patients who were injured at the middle age (eg, patients with injuries caused by a fall) increases. Older spinal-cord-injured patients have less flexibility in ROM, a condition which will become worse as a result of the initial bed rest needed to repair the injured spine.

Krause $^{10}$ reported that the odds of musculoskeletal conditions (curvature of the spine and major contractures) were more than 2.5 times higher for the groups of participants that were 20 or more years post-injury when compared with individuals who had been injured less than a decade. This significant tendency applies not only to the joints of the extremities, but also to the thorax and spine. ${ }^{11-14}$ More cases must be investigated before this tendency can be further elucidated.

\section{Conclusions}

Differences between traumatic tetraplegic patients (C6 complete injury) who could roll and those who could not roll were investigated using 3-dimensional measurement.

Rolling requires greater and adequate flexibility in the back of tetraplegic patients. We conclude that it would be helpful to take into consideration the condition of the subject's joint flexibility, not only in the extremities but also in the spine, when we evaluate the goal of rehabilitation.

\section{References}

1 Schonherr MC et al. Functional outcome of patients with spinal cord injury: rehabilitation outcome study. Clin Rehabil 1999; 13: $457-463$.

2 Yoshimura $\mathrm{O}$ et al. Possibility of independence in ADL (Activities of Daily Living) for the patients with cervical spinal cord injuries: An Evaluation based on the Zancolli Classification of residual arm. Hiroshima J Med Sci 1998; 47: 57-62.

3 Marino RJ, Goin JE. Development of a short-form Quadriplegia Index of Function scale. Spinal Cord 1999; 37: 289-296.

4 Fujiwara T et al. Relationship between shoulder muscle strength and functional independence measure (FIM) score among C6 tetraplegics. Spinal Cord 1999; 37: 58-61.

5 Nakayama T. Measurement Using a Magnetic Tracking Device. Rigaku ryohogaku 1998; 25: 255-257.

6 Tanaka S et al. Motion Analysis of Rolling. Journal of Hiroshima Physical Therapy 1999; 8: 6-9.

7 Lasfargues JE et al. A model for estimating spinal cord injury prevalence in the United States. Paraplegia 1995; 33: 62-68.

8 McGlinchey Berroth R et al. Late-life spinal cord injury and aging with a long term injury: characteristics of two emerging populations. Spinal Cord 1995; 18: 183-193.

9 Yarkony GM et al. Rehabilitation outcomes in C6 tetraplegia. Paraplegia 1988; 26: $177-185$.

10 Krause JS. Aging after spinal cord injury: an exploratory study. Spinal Cord 2000; 38: 77-83.

$11 \mathrm{Lim}$ TH et al. A noninvasive, three-dimensional spinal motion analysis method. Spine 1997; 22: $1996-2000$.

12 Pearcy MJ, Hindle RJ. New method for the non-invasive threedimensional measurement of human back movement. Clin Biomech 1989; 4: 73-79.

13 Nakajima A, Honda S. Physical and social condition of rehabilitated spinal cord injury patients in Japan: a long-term review. Paraplegia 1988; 26: $165-176$.

14 Suyama $\mathrm{T}$ et al. Rehabilitation of spinal cord injury in the national rehabilitation center for the disabled of Japan: profile of a spinal service. Spinal Cord 1997; 35: 720-724. 